\title{
Desarrollo y Libertad: Aplicación al proyecto de desarrollo de liderazgo de mujeres Aymaras en Puno (Perú)
}

\author{
FRANCIS CASTRO-MELO ${ }^{a}$, SUSANA SASTRE-MERINO ${ }^{b}$ \\ a Dirección General de Programas Especiales de la Presidencia, Ministerio de la Presidencia, Avda. \\ México, esquina Leopoldo Navarro, 102005 Santo Domingo, D.N., República Dominicana. E-mail: \\ franciscastro@hotmail.es \\ b Universidad Politécnica de Madrid, E.T.S. de Ingeniería Agronómica, Alimentaria y de Biosistemas, \\ Avda. Puerta de Hierro, 2, 28040 Madrid, España.E-mail: susana.sastre@upm.es
}

\begin{abstract}
RESUMEN
Desde la introducción del concepto de Development as Freedom, formulado por Amartya Sen, los métodos de evaluación del desarrollo se han enriquecido sustancialmente. A partir de este concepto, esta investigación presenta un desarrollo conceptual de los tipos de libertades; así, como una propuesta de jerarquización de las principales restricciones incidentes en el desarrollo; siendo el propósito fundamental, analizar el proceso de expansión de las libertades económicas, resultantes del proyecto: "Desarrollo de liderazgo de mujeres Aymaras en comunidades de Puno, Perú”. El estudio describe cómo el proceso de desarrollo de capacidades, en conjunción con elementos valorados, culmina en la expansión de las libertades económicas de las beneficiarias.

Palabras clave: Libertad, capacidad, funcionamiento, economía del desarrollo.
\end{abstract}

\section{Development as Freedom: Application to a Leadership Development Project of Aymara Women in Puno (Peru)}

\begin{abstract}
Since the introduction of the concept of Development as Freedom, developed by Amartya Sen, the methods of evaluation of development have substantially been enriched. From this concept, this research presents a conceptual development of the kinds of freedoms; as well as a proposal for prioritization of major restrictions affecting development. The main purpose is to analyze the process of expansion of economic freedoms resulting from the project: "Development of Aymara women leadership in communities of Puno, Peru". The study describes how the process of capacity building, in conjunction with motivational elements, culminates in the expansion of economic freedoms of the beneficiaries.
\end{abstract}

Keywords: Freedom, Capabilities, Functioning, Development Economics.

Clasificación JEL: O1, O2

Artículo recibido en abril de 2016 y aceptado en diciembre de 2016

Artículo disponible en versión electrónica en la página www.revista-eea.net, ref. ə-35110 


\section{INTRODUCCIÓN}

Para el Premio Nobel de economía 1998, Amartya Kumar Sen, el desarrollo puede concebirse, como un proceso de expansión de las libertades que disfrutan los individuos. La noción del enfoque de Sen, plasmado en su obra Development as Freedom se sustenta sobre la base de que el desarrollo debe ser evaluado en términos de... "la expansión de las 'capacidades' (capabilities) para llevar a cabo el tipo de vida que las personas valoran y que tienen razones para valorar" (Sen, 1999, p. 19). Según Sen, una capacidad refleja lo que una persona puede hacer y ser, independiente de que decida realizarlo. En consecuencia, las capacidades reflejan la libertad de una persona para elegir entre diferentes formas de vida (Nussbaum \& Sen, 1996).

En conjunción con lo antes escrito, la capacidad de una persona puede ser expresada a través de diferentes combinaciones de funcionamientos (functioning) (Montuschi, 2008) que puede conseguir una persona durante su vida. Es decir, la capacidad es la libertad fundamental para conseguir las distintas combinaciones de funcionamientos, o en términos menos formales, la libertad para lograr los diferentes estilos de vida que la persona valora (Sen, 1999).

El concepto de capacidades mantiene una estrecha vinculación con el de funcionamientos; sin embargo, los funcionamientos son independientes de las capacidades. Sen, define los funcionamientos como estado o acciones que una persona puede valorar. Los funcionamientos valorados pueden ir desde los elementales, como comer bien y no padecer enfermedades evitables, hasta actividades o estados personales muy complejos, como ser capaces de participar en la vida de la comunidad o respetarse a uno mismo (Cenci, 2011). La diferencia fundamental entre los funcionamientos y las capacidades, es que una persona puede poseer unas capacidades (disfrutar de ciertas libertades), pero puede decidir si llevarlas a cabo o no (Fernández, et al., 2012) dependiendo de los funcionamientos que pretenda alcanzar según su criterio.

La capacidad de una persona es dependiente de un conjunto de factores, que incluye las características personales y se complementa a través de los arreglos sociales (Nussbaum \& Sen, 1996). Sen (1999) nos enseña con su concepción de las capacidades que para explicar en su totalidad la libertad de un individuo se debe ir más allá de las capacidades de la vida personal, analizando el conjunto de objetivos de la persona, así como las metas sociales que tienen un vínculo directo con su propia vida.

Por el simple hecho de que la evaluación de la libertad trascienda la vida personal, pareciera que ésta solo se puede evaluar en términos colectivos. Sen resuelve este aparente conflicto con la introducción del Enfoque de Capacidades (EC), cuyo objeto esencial es valorar el bienestar individual de las personas, en su entorno institucional, así como el diseño de políticas o propuestas de cambios sociales (Robeyns, 2005). 
Lo fundamental del EC es que proporciona un conjunto de conceptos bastante útiles para evaluar el desarrollo. En consonancia, el EC permite contrastar los niveles de desarrollo -resultantes de una intervención- a través de la evaluación de las libertades individuales y colectivas, amparado fundamentalmente, en el uso de los conceptos de capabilities, functioning, y capacidad de agencia. En este sentido, Sen (1995, p.33) establece que "la capacidad es un reflejo de la libertad para alcanzar funcionamientos valiosos”.

Esta nueva visión introducida por Sen, amplió las nociones del desarrollo más allá del ingreso personal, el crecimiento del Producto Nacional Bruto (PNB) o el grado de industrialización de una sociedad en particular, adentrándose en discusiones menos abordadas por la economía ortodoxa, rescatando elementos de la economía política e introduciendo en el análisis un matiz de corte filosófico. El EC se centra pues en ampliar las libertades de las personas y no en favorecer únicamente el crecimiento económico.

Para complementar el EC, Sen utiliza el concepto ortodoxo de mercado, en el sentido de que reivindica una visión del mercado como lugar de intercambio, similar a la que tenían los clásicos como Adam Smith; aunque, en su concepción, tiene un rol un tanto diferenciado al de la teoría clásica, pues reivindica el papel de las instituciones como reguladoras del mercado. En su cosmovisión del mercado, Sen rescata, a su entender, su rol menos reconocido, a saber, su capacidad como "medio" de ampliación de las libertades. Desde su perspectiva, los mercados juegan un papel preponderante como mecanismo propagador de libertades, asegurando que existen buenas razones para comprar y vender, para participar en el mercado de trabajo, para intercambiar y para tratar de llevar una vida que pueda florecer gracias a las transacciones, y en seguida asiente... "negar esa libertad en general sería en sí mismo un fracaso de una sociedad”... al considerar el papel omnipresente que actualmente tienen las transacciones en la vida moderna.

Otro de los grandes aportes de Sen al EC, es la introducción del concepto de agente o capacidad de agencia, entendido como: "la habilidad que tiene una persona para conseguir las metas y valores que considera importantes" (Sen, 1999, p. 234), mismo que aplica para estudiar el rol preponderante que han venido desempeñando las mujeres en la construcción del desarrollo. Según Sen (1999, p.233) ... "las mujeres han dejado de ser receptores pasivos de la ayuda destinada a mejorar su bienestar y son vistas, tanto por los hombres como por ellas mismas, como agentes activos de cambio"... es decir, visualiza a las mujeres como promotoras de las transformaciones que se suscitan hoy por hoy en la sociedad. Los objetivos han cambiado, y se han ampliado poco a poco: se ha dejado de centrar la atención en el bienestar y se ha incorporado y subrayado el papel activo de la mujer (Sen, 1999). 
Fomentar la capacidad de actuar de las mujeres, es uno de los mayores retos del desarrollo desde la perspectiva de las libertades, puesto que supone uno de los principales elementos que intervienen en los cambios sociales y económicos.

Entre los factores que intervienen en el proceso de desarrollo, está la necesidad de educación de las mujeres, las oportunidades de empleo y su desempeño en el mercado de trabajo (Urquijo, 2007). Mediante el fomento de las libertades se contribuye a la capacidad de acción de las mujeres, al ampliar sus oportunidades.

En el concepto de agente juega un papel central la faceta de compromiso, que es la base desde donde se pueden lograr los propósitos, ya que están directamente relacionados con los elementos motivacionales de valores compartidos y la interacción con otros. Tanto la capacidad de actuar como el compromiso, responden a la libertad que poseen los sujetos de perseguir como agentes responsables cualquier meta o valor que consideren importantes en sus vidas (Pallas, 2008).

Teniendo en cuenta lo anterior, uno de los propósitos de este artículo es realizar un desarrollo conceptual sobre los tipos de libertades concebidos por Sen, y presentar una propuesta de jerarquización de las restricciones a las libertades que más afectan al desarrollo; siendo el propósito cardinal, analizar bajo una perspectiva del Enfoque de las Capacidades (capabilities approach), la expansión de las libertades económicas, resultantes de la implementación del proyecto: "Desarrollo de liderazgo de mujeres Aymaras en comunidades de Puno, Perú”. La investigación analiza cómo a través del proceso de desarrollo de capacidades (capabilities) en conjunción con los functioning y la capacidad de agencia de la Coordinadora de Mujeres Aymaras (CMA) pasa de ser de una agrupación cuyo objeto fundamental era alcanzar libertades constitutivas, a ser una organización empresarial que alcanzó mayores niveles de libertades instrumentales (libertades económicas), repercutiendo en la mejora de la calidad de vida, tanto de las beneficiarias, como de sus familiares.

Para llevar a cabo el análisis, la metodología aplicada consistió en analizar el estudio de caso desde el Enfoque de Capacidades de Sen. Para ello se contó como fuente de información secundaria con cuestionarios y entrevistas en profundidad obtenidos por un investigador del grupo de investigación GESPLAN de la Universidad Politécnica de Madrid desde el año 2011 hasta 2014. El objetivo de dicha investigación fue analizar el desarrollo de capacidades a través de un proyecto de desarrollo rural, medido a través de las competencias de las participantes. A partir de la información disponible, se hizo un trabajo de reclasificación de las competencias identificadas. Para ello, se partió del agrupamiento ya realizado en el estudio previo, según las dimensiones de las competencias para la dirección de proyectos de la International Project Management Association (IPMA): técnicas, contextuales y de comportamiento. Se analizaron las similitudes de este enfoque con el concepto de libertad acuñado 
por Sen y como resultado se reclasificaron las competencias en tres categorías: capacabilities, functioning y capacidad de agencia.

Los datos analizados demuestran que el proyecto contribuyó a la expansión de las libertades de las beneficiarias, a través de la promoción y construcción de capacidades. El caso de estudio también indica que el compromiso con el colectivo como reflejo del estado alcanzado y la capacidad de agencia, jugaron un papel preponderante, debido a que las libertades facilitadas por la intervención fueron bien aprovechadas.

\section{CONCEPTUALIZACIÓN Y JERARQUIZACIÓN DE LIBERTADES}

A juicio de Sen (1999, p. 55), la expansión de la libertad es el "fin primordial y el medio principal” del desarrollo. En este sentido, hace una primera distinción entre dos grandes grupos o tipos de libertades, en función de uno u otro, a saber: libertades constitutivas y libertades instrumentales. Al referirse a la libertad como "fin" distingue el papel constitutivo, mientras que, si hace referencia a la libertad como "medio" alude el papel instrumental.

Haciendo una diferenciación conceptual, el papel constitutivo apunta al reconocimiento de las libertades fundamentales para el enriquecimiento de la vida humana (Sen, 1999, p. 55). Entre las libertades constitutivas se encuentran algunas capacidades elementales como, por ejemplo, poder evitar privaciones como la inanición, desnutrición, morbilidad evitable, mortalidad prematura o gozar de capacidades como leer, escribir y calcular, libertad de participación política, libertad de expresión y participación. Desde la perspectiva constitutiva, el desarrollo precisa de la expansión de estas y otras libertades básicas. El desarrollo es el proceso de expansión de las libertades humanas y su evaluación ha de inspirarse en esta consideración (Sen, 1999, p. 56).

Por su parte, las libertades instrumentales son las que contribuyen, directa o indirectamente, a la libertad general que tienen los individuos para vivir como les gustaría. Las libertades instrumentales constituyen el foco principal de atención en la obra de Sen.

La diversidad de libertades instrumentales es bastante grande; sin embargo, Sen (1999, p. 57) considera útil identificar cincos tipos distintos:

- Las libertades políticas, concebidas en un sentido amplio (incluido los llamados derechos humanos), y referidas a las oportunidades que tienen los individuos para decir quién los debe gobernar y con qué principios, y comprenden también la posibilidad de investigar y criticar a las autoridades, la libertad de expresión política y prensa sin censura, la libertad para elegir entre partidos políticos, entre una larga lista más. 
- Las libertades económicas, refiriéndose a la libertad de los individuos, para consumir, producir o realizar intercambios. Los derechos económicos que tienen las personas dependen de los recursos que posea, a los que tenga acceso, así como de las condiciones de intercambio, como los precios relativos y el funcionamiento de los mercados.

- Las oportunidades sociales, que se refieren a los servicios que deben ser provistos por los gobiernos, como los sistemas de educación, sanidad, etc., que tiene la sociedad.

- Las garantías de transparencia que se refieren a la "necesidad de franqueza" que pueden esperar los individuos: la libertad para interrelacionarse con la garantía de divulgación de información y de claridad. Esta garantía desempeña un claro papel instrumental en la prevención de la corrupción, de la irresponsabilidad financiera y de los tratos poco limpios.

- La seguridad protectora necesaria para proporcionar una red de protección social que impida que la población afectada caiga en la mayor de las miserias, la inanición o la muerte.

Estas libertades instrumentales mejoran directamente las capacidades de los individuos, pero también se complementan y pueden reforzarse mutuamente (Sen, 1999). Por ejemplo, el aumento en la renta personal tiende a reforzar a que se puedan financiar otras actividades como el acceso a servicios sociales como educación y sanidad, entre otros.

Los directores de carteras, programas y proyectos de desarrollo, las ONGD, así como los gestores de políticas públicas, al momento de elaborar los planes de intervención precisan de mecanismos que les permitan determinar las principales barreras que se contraponen al desarrollo a través de la supresión de libertades. En palabras de Sen (1999, p. 19) el desarrollo... "exige la eliminación de las principales fuentes de privación de libertad". Tal como se ha planteado, Sen distingue entre algunos tipos de libertades; empero, no de manera sistemática (Gough, 2007). Debido a que puede ser bastante útil para algunos ejercicios evaluativos, identificar un subconjunto de capacidades crucialmente importantes (Nussbaum \& Sen, 1996), se elaboró un ejercicio de ordenación sistemática de factores que atentan contra las libertades y se clasificaron en función de si esos factores restringen capacidades básicas o aquellas que contribuyen a vivir mejor, ordenadas de mayor a menor nivel de importancia.

Se entiende por capacidades básicas aquellas que hacen referencia a ciertos funcionamientos crucialmente importantes, hasta ciertos niveles adecuadamente mínimos (Nussbaum \& Sen, 1996). Estas libertades están inminentemente relacionadas con las antes mencionadas libertades fundamentales como la habilidad para tener una vida larga y próspera, el poder leer, escribir y evitar enfermedades, a las que además se le suman la ausencia de violencia física y 
psicológica; conflictividad civil, étnica y religiosa; delincuencia común y organizada; violencia doméstica; discriminación de género; mano de obra forzada a niños y adultos; represión de la capacidad cognitiva, entre otras.

Cuando se hace alusión a la clasificación para vivir mejor, se alude a las antes mencionadas libertades instrumentales; es decir, a las libertades que contribuyen a que el individuo viva como le gustaría vivir.

Tabla 1

Propuesta de jerarquización de las principales restricciones en función de su incidencia en el proceso de desarrollo

\begin{tabular}{|c|c|c|c|}
\hline $\begin{array}{l}\text { Tipos de libertad de } \\
\text { acuerdo a su nivel de } \\
\text { importancia } \\
\text { (de mayor a menor) }\end{array}$ & Clasificación & Papel & $\begin{array}{c}\text { Cómo caracterizar su incidencia como elemento } \\
\text { supresor del desarrollo }\end{array}$ \\
\hline $\begin{array}{l}\text { 1. Libertades } \\
\text { fundamentales }\end{array}$ & Básicas & Constitutiva & $\begin{array}{l}\text { Inanición, desnutrición, morbilidad evitable, mortalidad } \\
\text { prematura, capacidad de leer, escribir, calcular, } \\
\text { participación política, libertad de expresión, otras (...) }\end{array}$ \\
\hline 2. Seguridad personal & Básicas & Constitutiva & $\begin{array}{l}\text { Ausencia de violencia física y psicológica (violación de } \\
\text { la soberanía; conflictos civiles; étnicos y religiosos; } \\
\text { delincuencia común y organizada; violencia } \\
\text { doméstica; discriminación de género; mano de obra } \\
\text { forzada a niños y adultos; represión de la capacidad } \\
\text { cognitiva y emocional) }\end{array}$ \\
\hline $\begin{array}{l}\text { 3. Libertades } \\
\text { económicas }\end{array}$ & Para vivir mejor & Instrumental & $\begin{array}{l}\text { Libertad de consumir, producir y de participar en los } \\
\text { mercados a través de las transacciones }\end{array}$ \\
\hline 4. Libertades políticas & Para vivir mejor & Instrumental & $\begin{array}{l}\text { Garantías de elegir y ser elegido, libertad de expresión } \\
\text { política y prensa sin censura, libertad de elegir entre } \\
\text { diferentes partidos políticos, otras (...) }\end{array}$ \\
\hline $\begin{array}{l}\text { 5. Oportunidades } \\
\text { sociales }\end{array}$ & Para vivir mejor & Instrumental & $\begin{array}{l}\text { Libertad de acceso a educación y servicios sanitarios, } \\
\text { seguridad jurídica, acceso a vivienda, cobertura de } \\
\text { pensiones y prestaciones (...) }\end{array}$ \\
\hline $\begin{array}{l}\text { 6. Garantías de } \\
\text { transparencia }\end{array}$ & Para vivir mejor & Instrumental & $\begin{array}{l}\text { Libertad de interrelación, garantías de divulgación y } \\
\text { claridad, garantías de transparencia, otras (...) }\end{array}$ \\
\hline $\begin{array}{l}\text { 7. Seguridad } \\
\text { protectora }\end{array}$ & Para vivir mejor & Instrumental & $\begin{array}{l}\text { Prestaciones por desempleo, ayudas económicas a } \\
\text { indigentes, mecanismos ad hoc para aliviar las } \\
\text { hambrunas, empleo público de emergencia, otras (...) }\end{array}$ \\
\hline $\begin{array}{l}\text { 8. Seguridad } \\
\text { ambiental }\end{array}$ & Para vivir mejor & Instrumental & $\begin{array}{l}\text { Equilibrio medioambiental y sostenibilidad del } \\
\text { desarrollo (Deterioro de los ecosistemas; mal uso y } \\
\text { sobreexplotación de los recursos no renovables; } \\
\text { desastres naturales; contaminación) (...) }\end{array}$ \\
\hline
\end{tabular}

Fuente: Elaboración propia a partir de: Sen, A. K., (1999). Desarrollo y Libertad; Informe de Desarrollo Humano 1994; L. Doyal e I. Gough, (1991). A Theory of Human Need; Pérez, C. \& Areizaga, M., (2012.) Seguridad humana.

En adición a las libertades fundamentales antes descritas y las cinco libertades instrumentales propuestas por Sen, se han sugerido otras dos, extraídas del concepto de seguridad humana, que aparece por primera vez en el Informe de Desarrollo Humano de 1994. Estas son: (1) la seguridad personal, que se refiere a la capacidad del individuo de vivir libre de violencia física (es decir, ausente de elementos violatorios de la soberanía; conflictos civiles; étnicos y religiosos; delincuencia común y organizada; violencia doméstica; discriminación de género; 
mano de obra forzada a niños y adultos, etc.) (Pérez \& Areizaga, 2007), a la que se le suma, la violencia psicológica, entendida como represión de la capacidad cognitiva y emocional (Gough, 2007); y (2) la seguridad ambiental, que se refiere a la capacidad del ser humano de vivir en equilibrio medioambiental y en sostenibilidad con el desarrollo (para mejor ilustración ver Tabla 1) (Pérez \& Areizaga, 2007). Ambas libertades se consideran relevantes por el carácter de complementariedad que podrían impregnarle al caso de estudio, y porque se entiende que por sí solas, las libertades descritas por Sen, no ofrecen una visión que pudiese abarcar todo el espectro de aplicación del caso de estudio.

\section{CONTEXTO DEL CASO DE ESTUDIO: DESARROLLO DE LIDERAZGO DE MUJERES AYMARAS EN COMUNIDADES DE PUNO (PERÚ)}

En el siguiente apartado se contextualiza la organización objeto de estudio. Se trata de una organización fundada en 1981 en comunidades de mujeres aymaras en Puno, Perú. El contexto institucional y de actuación del proyecto permite comprender las libertades existentes en esa organización y las actuaciones llevadas a cabo para acrecentar sus oportunidades.

\subsection{Contexto institucional: Coordinadora de Mujeres Aymaras (CMA)}

La Coordinadora de Mujeres Aymaras (CMA) es una organización fundada en 1981, en Juli, capital de la provincia de Chucuito, situada en la región Puno, Perú. Según (Yagüe \& Cazorla, 2013), en sus inicios tomo el nombre de “Coordinación Pastoral de Mujeres de la Prelatura de Juli”, cuyos objetivos iníciales eran la evangelización, la lucha por los derechos de la mujer, la lucha contra la violencia familiar, además de realizar actividades de catequesis, que posteriormente acondicionaron a la búsqueda del desarrollo personal, grupal, comunal, el fortalecimiento institucional, la mejora de la salud y la capacitación de sus integrantes en diferentes actividades.

Desde la década de 1990, la CMA ha interactuado con diversas instituciones externas, como Caritas, el Instituto de Educación Rural de Juli (IER) y la Vicaría de Solidaridad, entre otras, para la formación y capacitación en diferentes actividades (Sastre-Merino, et al., 2013). En 2006, la organización, a través del obispo de Juli, hizo el primer contacto con el Grupo de investigación en planificación y gestión sostenible del desarrollo rural-local, GESPLAN, de la Universidad Politécnica de Madrid, que se encontraba trabajando en áreas adyacentes. Después de una serie de talleres iniciales, el proyecto inició en 2008 y la organización fue establecida legalmente bajo el nombre "Coordinadora de Mujeres Aymaras” (Yagüe \& Cazorla, 2013). 
En el 2007 el grupo GESPLAN-UPM inicia un diagnóstico de la zona de actuación, para determinar el tipo de acciones que se podrían tomar para responder a la petición de la CMA (Sastre-Merino 2014), que en 2008 culminó con la elaboración del proyecto de trabajo, bajo el título "Desarrollo de las capacidades de liderazgo de la mujer en las comunidades aymaras de Puno (Perú)" (Yagüe \& Cazorla, 2013), siendo aprobado dos años más tarde.

El principal objetivo del proyecto fue: "Incrementar la capacidad técnica y empresarial de las mujeres microempresarias de las comunidades aymaras de la región de Puno (Perú)" (Cazorla, et al., 2010). Este objetivo responde a una de las mayores demandas detectadas de las mujeres rurales, que es tener un mayor acceso y control sobre los recursos productivos (Sastre-Merino, 2014).

Como elemento neurálgico del proceso de diseño, en los trabajos realizados durante el año 2007, se identificaron las actividades de producción artesanal de textiles de calidad, las producciones orgánicas, la transformación de lácteos y la crianza de animales domésticos para el autoconsumo y la venta local, como actividades en que las beneficiarias podían involucrarse para mejorar su nivel de vida, de sus familias y de sus comunidades (Yagüe \& Cazorla, 2013). Inicialmente las líneas estuvieron encaminadas al emprendimiento de acciones agropecuarias; más tarde, al desarrollo de artesanía textil, convirtiéndose en la línea de acción de mayor éxito del proyecto (Sastre-Merino, 2014).

\subsection{Población objetivo y área de estudio}

La población objetivo de la aplicación del caso de estudio, está conformada por mujeres de las comunidades rurales aymaras en las provincias de Moho, Huancané, Puno, Chucuito, Juli, Collao y Yunguyo (Cazorla, et al., 2010) en el Perú, representando el grupo a priorizar por las actividades y los agentes participantes en el proyecto (Yagüe \& Cazorla, 2013). Actualmente la organización está conformada por 320 artesanas, divididas en 21 grupos, distribuida espacialmente en tres zonas: Norte, Centro y Sur (Sastre-Merino, 2014).

\section{METODOLOGÍA}

En esta sección se describe la metodología empleada, que utiliza como soporte teórico el Enfoque de Capacidades y los conceptos de capabilities, functioning y capacidad de agencia para la evaluación de la expansión de las libertades económicas de las beneficiarias resultantes de la intervención.

La investigación usó como soporte empírico las bases de datos elaboradas por GESPLAN-UPM para analizar el desarrollo de capacidades resultantes de la intervención a través de las competencias de las participantes. Dichas bases de datos contenían la información recogida mediante tres cuestionarios y entrevistas 
personales y organizada en tres grandes bloques: capacidades técnicas (habilidades y conocimientos necesarios para gestionar el proyecto eficazmente), contextuales (referidas a las habilidades necesarias para que las partes involucradas interactúen con el contexto del proyecto y con la organización permanente) y de comportamiento (relacionadas con las relaciones personales que se producen entre las personas y grupos involucrados en el proyecto) de acuerdo con los estándares de competencias de la NCB de la International Project Management Association (IPMA), lo que simplificó su explotación.

El primero de los cuestionarios tenía como objetivo evaluar el desarrollo de las capacidades individuales. Se realizaron 144 cuestionarios estructurados en seis bloques temáticos (emigración; infraestructura y servicios; aspectos productivos; proyecto; desarrollo y bienestar de la comunidad), equiparables al 45 por ciento del total de mujeres asociadas a la CMA (en total 320 mujeres), levantados en los meses de abril y mayo de 2010. La herramienta, previo a su aplicación, fue validada por un panel de expertos en proyectos de desarrollo rural y por un grupo de mujeres pertenecientes a la junta directiva de la CMA. A partir de la información de este cuestionario, se reconfiguró y procesó la información para la realización de un análisis inicial de las capacidades de la CMA (Sastre-Merino, 2014).

El segundo cuestionario fue aplicado a una muestra de 106 mujeres aymaras, (equiparable al 33 por ciento del total) que participan de manera activa en la CMA. En él se evaluó el desarrollo de capacidades a nivel individual. La aplicación de este cuestionario fue segmentada en dos fases: una primera realizada en el 2011 y la segunda en 2012 (Sastre-Merino, 2014).

Para la evaluación del desarrollo de capacidades a nivel de la organización, se planteó un tercer cuestionario, que se orientó a las líderes de la junta directiva de la CMA (3 líderes), así, como al equipo planificador (5 personas del equipo técnico), debido a la visión holística que tienen sobre la evolución de las capacidades de la organización. La encuesta se realizó en 2012 y se aplicó a todo el equipo técnico, formado por cinco personas, y las tres líderes principales de la junta directiva (Sastre-Merino, 2014).

Finalmente, la información cuantitativa obtenida mediante los cuestionarios, fue complementada con información cualitativa, lo que permitió comprender y profundizar algunos temas (Niremberg, 2005) levantados en los cuestionarios cuantitativos. Esta información fue recopilada mediante entrevistas en profundidad, así, como a través de entrevistas semiestructuradas, realizadas a personas consideradas “claves” en el proceso de dirección del proyecto (SastreMerino, 2014).

Bajo la premisa sustentada por Sen, en cuanto a que la libertad refleja lo que una persona puede hacer o ser independientemente de que decida realizarlo, se seleccionaron aquellas preguntas de los cuestionarios que hacen alusión, directa 
o indirecta, a la variación en los niveles tanto de capabilities, functioning y capacidad de agencia resultantes de la intervención. Las respuestas se agruparon para su análisis en tres grupos: el primero, las "capabilities", integrado por las preguntas de las dimensiones técnica, contextual y de comportamiento, que en últimas permiten determinar las capacidades desarrolladas por las beneficiarias. El segundo bloque queda conformado por los functioning. Para la valoración de los functioning, se procedió mediante análisis del aprovechamiento de la dimensión contextual. A los fines, solo se midieron las funciones más elementales ponderadas por parte de las beneficiarias, a través del grado de satisfacción con el ingreso percibido por pieza y el acceso a nuevos nichos de mercado, que en últimas refleja la libertad económica de poder realizar mayor número de transacciones. El tercer bloque analiza la capacidad de agencia a través de las dimensiones técnicas, contextuales y de comportamiento, agrupando las preguntas alusivas a variaciones en el liderazgo, la asunción de funciones administrativas y financieras propias de la CMA y las consecuencias producto del incremento de los niveles de ingreso de las beneficiarias (ver Tabla 2).

\section{Tabla 2}

Clasificación metodológica de los bloques, dimensiones e ítems.

\begin{tabular}{|c|c|c|}
\hline Bloque & Dimensión & Ítems \\
\hline \multirow[t]{3}{*}{ Capabilities } & Técnica & $\begin{array}{l}\text { 1. Capacidad para tejer; } \\
\text { 2. Cómo ha mejorado el proyecto sus capacidades para el } \\
\text { desarrollo de sus productos; } \\
\text { 3. Qué han aprendido con su pertenencia a la organización } \\
\text { (técnicas); } \\
\text { 4. Uso nuevos materiales; } \\
\text { 5. Capacidad para realizar el control de calidad. }\end{array}$ \\
\hline & Comportamiento & $\begin{array}{l}\text { 1. Qué han aprendido con su pertenencia a la organización } \\
\text { (liderazgo y aprender a compartir). }\end{array}$ \\
\hline & Contextual & $\begin{array}{l}\text { 1. Canales de comercialización de los productos } \\
\text { destinados al mercado. }\end{array}$ \\
\hline \multirow{3}{*}{ Capacidad de Agencia } & Técnicas & $\begin{array}{l}\text { 1. Encargarse de las operaciones administrativas y } \\
\text { contables de la oficina. }\end{array}$ \\
\hline & Contextual & $\begin{array}{l}\text { 1. Aumento del número de transacciones (aumento del } \\
\text { ingreso personal y familiar); } \\
\text { 2. Aumento del número de transacciones (acceso a nuevos } \\
\text { nichos de mercado). }\end{array}$ \\
\hline & Comportamiento & $\begin{array}{l}\text { 1. Preferencia por el trabajo en equipo (Apoyarse y } \\
\text { aprender las unas de las otras); } \\
\text { 2. Nivel de participación en las decisiones de la } \\
\text { organización. }\end{array}$ \\
\hline Functioning & Contextual & $\begin{array}{l}\text { 1. Grados de satisfacción con el ingreso percibido por } \\
\text { pieza; } \\
\text { 2. Aumento del número de transacciones (acceso a nuevos } \\
\text { nichos de mercado); } \\
\text { 3. Impacto del proyecto en sus hogares (mejora en la } \\
\text { alimentación y educación de sus hijos). }\end{array}$ \\
\hline
\end{tabular}

Fuente: Elaboración propia. 


\section{RESULTADOS}

En términos generales, el proceso de expansión de las libertades, es resultado de la potenciación de las capabilities, que conjuntamente con los Functioning y la capacidad de agencia, contribuyeron a la expansión de las libertades económicas de las beneficiarias.

\subsection{Valoración de las capacidades (capabilities), los funcionamientos (functioning) y la capacidad de agencia}

Para la valoración de las capacidades resultantes de la intervención, se realizaron análisis basados en el EC, que permitieron la descripción de la perspectiva, tanto de las beneficiarias como del equipo de dirección, relativo a la variación en sus habilidades.

El análisis basado en el EC, muestra que inicialmente los objetivos de la organización se centraban en la expansión de libertades constitutivas (libertades fundamentales y seguridad personal) tales como: la evangelización, la lucha por los derechos de la mujer y la lucha contra la violencia familiar, entre otras. Posteriormente, mediante el incremento de las capacidades técnicas y contextuales, y en menor rango las capacidades de comportamiento, acondicionaron su accionar a la búsqueda de libertades de orden instrumental (como el desarrollo personal, grupal, comunal, el fortalecimiento institucional, la mejora de las condiciones de salubridad y la capacitación de sus integrantes en diferentes actividades), fundamentándose en la mejora de la actividad empresarial, lo que produjo un incremento del volumen transaccional (ver Figura 1).

Figura 1

Proceso de transición entre las libertades constitutivas a libertades económicas

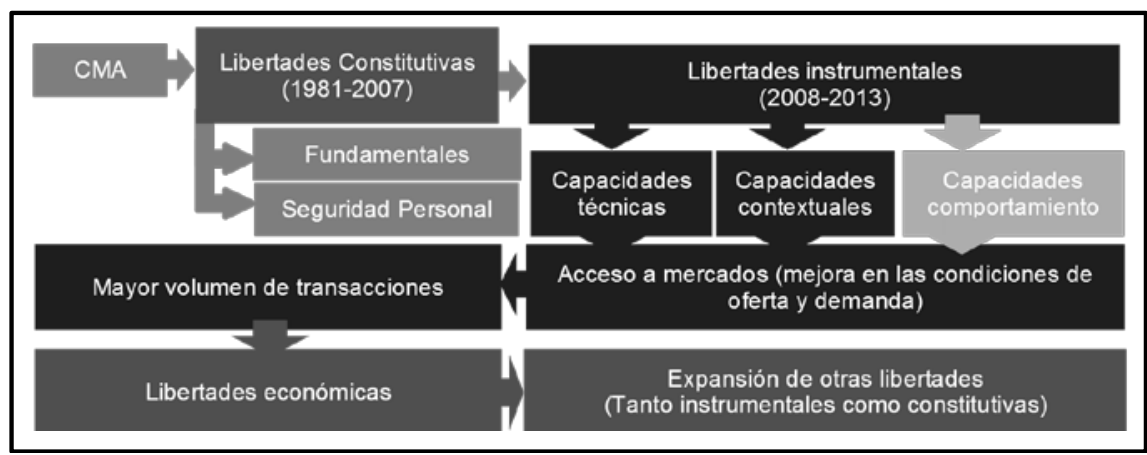

Fuente: Elaboración propia.

Los resultados muestran que uno de los aportes de mayor relevancia realizados por la intervención, como parte del proceso de desarrollo de las capabilities, los Functioning y la capacidad de agencia, fue que les permitió a 
las beneficiarias la expansión de un tipo de libertad instrumental que Sen (1999, p. 58) describe como "libertades económicas", a través de la optimización de las cadenas de valor de los productos y su adecuada articulación en el mercado (Yagüe \& Cazorla, 2013).

Previo a la intervención, sus productos se comercializaban, en un $88 \%$ de los casos, a través de intermediarios. Además, mediante el proyecto las mujeres recibieron un precio más alto por sus piezas de artesanía, que en algunos casos triplicaba el precio anterior (Sastre-Merino, et al., 2013). Esto se evidencia por el grado de satisfacción de las beneficiarias con el ingreso percibido por pieza, que alcanzó niveles del $80 \%$. La simple posibilidad de aumentar su nivel de ingreso, permitió acrecentar sus opciones, incrementando su capacidad de elección (capabilities) y las alternativas de los elementos que considera crucialmente importantes (functioning), como destinar el incremento en sus ingresos a alimentarse mejor, invertir en su salud, su educación o las de sus familiares.

El proceso de expansión de las libertades económicas se trabajó fundamentalmente desde una perspectiva de mejora del nivel de las capacidades técnicas y contextuales de las beneficiarias. A continuación, se muestran los principales resultados asociados.

Como sustento empírico se muestra (en encuesta realizada en 2011) que el 94.3\% de las beneficiarias manifestó que había mejorado su capacidad técnica para tejer, producto de las capacitaciones recibidas. En la encuesta de 2012, se les pidió clasificar su grado de acuerdo con que el proyecto había contribuido a mejorar sus capacidades para desarrollar los productos, y en el $50 \%$ de los casos, las entrevistadas manifestaron estar bastante o muy de acuerdo, un $44 \%$ algo de acuerdo y solo un $6 \%$ en desacuerdo.

A las beneficiarias también se les preguntó si consideraban que habían aprendido algo valioso con su pertenencia a la organización, y en el 95\% de casos, las que respondieron que sí, relacionaron de manera espontánea su aprendizaje con las capacitaciones técnicas. El 5\% restante señaló otras como "las capacitaciones en liderazgo" y "aprender a compartir".

Un resultado bastante relevante derivado de la intervención y que potenció mayores rendimientos en las capacidades de las beneficiarias, es la inclusión en el equipo de proyectos de la ONG Diseño para el Desarrollo (DPD). DPD contribuyó al proceso de ampliación de las libertades de las beneficiarias, al proporcionarles conocimiento empresarial sobre el mercado, mediante el apoyo en el desarrollo de los diseños, así como asistencia técnica en el diseño de sus prendas (Sastre-Merino, Negrillo, \& Hernández, 2013), la utilización de las tallas del mercado, el etiquetado de las prendas y los datos que deben aparecer en las misma, ajuste de precios según coste y tiempo de trabajo, acceso al 
mercado por otros cauces que evite el abuso de los intermediarios, entre otras (Yagüe \& Cazorla, 2013).

Otra capacidad de índole técnica que ha quedado afincada con la intervención, es el uso de nuevos materiales como: la baby alpaca, la alpaca FS, las mezclas de alpaca con acrílico, el algodón en prendas de tejido a punto y el bordado sobre nuevas superficies como la bayeta natural, industrial, bayetilla, loneta, tela de algodón, entre otras innovaciones que han modificado las características de producción (Negrillo, et al., 2011), derivando en la ampliación del abanico de funciones posibles (Cenci, 2011) de la CMA, permitiéndoles acceder a nichos de mercado que antes no tenían, sin intermediación, con un mayor nivel de valor agregado en sus productos (mayor nivel de productividad) derivado del proceso de tecnificación.

Debido a que el mercado de destino de los productos es bastante exigente, las capacitaciones en términos de gestión de calidad jugaron un papel preponderante. Desde el 2009 al 2012, se realizaron varios talleres de calidad y de diseño con el objeto de mejorar y diversificar la producción. En 2010 se determinó que la capacidad para elaborar productos de calidad era de un 67\%, lo cual, a consideración de ellas mismas, era su mayor debilidad (Sastre-Merino, 2014). En 2012 se les preguntó sobre si habían mejorado su capacidad para realizar control de calidad, a lo que el $27 \%$ respondió que nada, un $30 \%$ algo, un $25 \%$ bastante y solo un $17 \%$ respondió que mucho. La intervención, se enfocó en potenciar el desarrollo de la artesanía textil -aprovechando la libertad económica que brindan los mercados- encaminando la organización por un proceso de mejora de la actividad empresarial.

Producto del efecto de la complementariedad de las libertades descrito por Sen, la expansión de las libertades económicas tiene repercusiones directas sobre las capacidades de los individuos, reforzando otros tipos de libertades. Esto se evaluó al preguntarle a las beneficiarias sobre cuál había sido el impacto del proyecto en sus hogares. La mayoría de las beneficiarias manifestó que se sienten apoyadas por sus familias y ven el proyecto como una oportunidad para mejorar la alimentación en sus hogares y la educación de sus hijos (SastreMerino, 2014). En palabras de una de éstas (Sastre-Merino, 2014, p. 267) "la CMA nos ayuda a tener más pedidos, a aumentar ingresos, para nuestras familias y para educación de nuestros hijos". Otra señora dijo "la CMA me gusta mucho porque me ha permitido pagar la entrada de mi casa"... esto demuestra las importantes repercusiones que pesan sobre las libertades económicas y la capacidad de agente de las beneficiarias en el entorno familiar, ya que les brinda mayores oportunidades (capabilities) para conseguir las metas y valores que considera importantes (Nussbaum \& Sen, 1996) como alimentar y educar a su prole (functioning). 
Un resultado muy vinculado a la capacidad de agente de las mujeres aymaras, y que además tiende a reforzar de manera transversal, tanto a las capabilities como a los Functioning, es el aumento sostenido en las capacidades de liderazgo de las beneficiarias relacionado al proceso productivo, especialmente entre las líderes del grupo, ya que han podido aprender dónde comprar la materia prima para la elaboración de los productos, han viajado a las exposiciones y se empiezan a involucrar en el trabajo de oficina, lo que les permitiría administrar el proyecto en el largo plazo (Sastre-Merino, et al., 2013).

Como resultado de este progresivo empoderamiento, no sólo se benefician las mujeres aymaras, sino toda su familia, y por ello se considera que se produce un liderazgo territorial, pues como se ha dicho, las mujeres indican que usan los ingresos para mejorar la calidad de vida de sus hijos (Sastre-Merino, 2014). Esto demuestra que la ampliación de libertades instrumentales, como son las libertades económicas, no solo mejora directamente las capacidades de los individuos, sino que también se complementan y tienden a reforzarse mutuamente; por tanto, las libertades económicas de las beneficiarias han de juzgarse no solo en función del aumento de la renta privada (valoración individual), sino también, en función de la expansión de ciertas libertades instrumentales, como son las oportunidades sociales (Sen, 1999).

Un resultado vinculado a la capacidad de agencia que resulta interesante observar, es el trabajo en equipo. En el 2010, el 85\% de las beneficiarias indicó una preferencia generalizada por trabajar reunidas para apoyarse y aprender unas de otras (Sastre-Merino, 2014). En 2012, se les preguntó sobre si había mejorado su valoración, a lo que el $25 \%$ respondió que no había mejorado en nada, un $9 \%$ bastante y un 54\% mucho. El comportamiento generalizado sobre el trabajo en equipo queda evidenciado a través de las palabras de una de las beneficiarias, al expresar: "hay que trabajar unidas. No quedarse el conocimiento para uno" (Sastre-Merino, 2014, p. 274). En este sentido presupone un razonamiento que incluye consecuencias para los demás, que puede ir más allá de la racionalidad o la conducta egoístas, en virtud de la simpatía, con la incorporación de otras motivaciones a la conducta humana, posibilitando incorporar a otros como parte de la valoración, elección y acción (Sen, 1986). En la entrevista realizada en el 2011-2012, se midió el grado de compromiso de las beneficiarias, a través de preguntas de categorías ordenadas tipo Likert. La primera hace alusión al "aumento de la participación en las decisiones de la organización” en donde solo el 16\% dijo haber participado siempre en el proceso de toma de decisiones.

\section{ANÁLISIS Y DISCUSIÓN}

A continuación se muestra cómo se funden los conceptos de capabilities, Functioning y libre agencia en torno a las libertades económicas. 
La ampliación de las libertades económicas de las beneficiarias, partió inicialmente de dos procesos: la ampliación de las capacidades técnicas y las capacidades de comportamiento. La expansión de las capacidades técnicas producto del proceso de tecnificación y las mejoras incorporadas en los procesos de elaboración de los productos (Yagüe \& Cazorla, 2013), así como el impulso en las capacidades de comportamiento generadas por la intervención (que tuvo efectos considerables en la generación de capacidades de liderazgo en las beneficiarias), devino en el incremento de la productividad laboral ( $\triangle \mathrm{PL})$. A su vez, el incremento en PL generó dos efectos en el mediano plazo: el incremento de la producción $(\Delta \mathrm{Y})$ aumentando la cantidad unitaria de producto por unidad de tiempo y bajo los estándares de calidad requeridos (eficacia), y la expansión de la curva de aprendizaje ( $\Delta \mathrm{CA}$ ) que tiene efectos en la reducción de costes (eficiencia) (ver Figura 2).

Figura 2

Proceso de ampliación de las libertades económicas

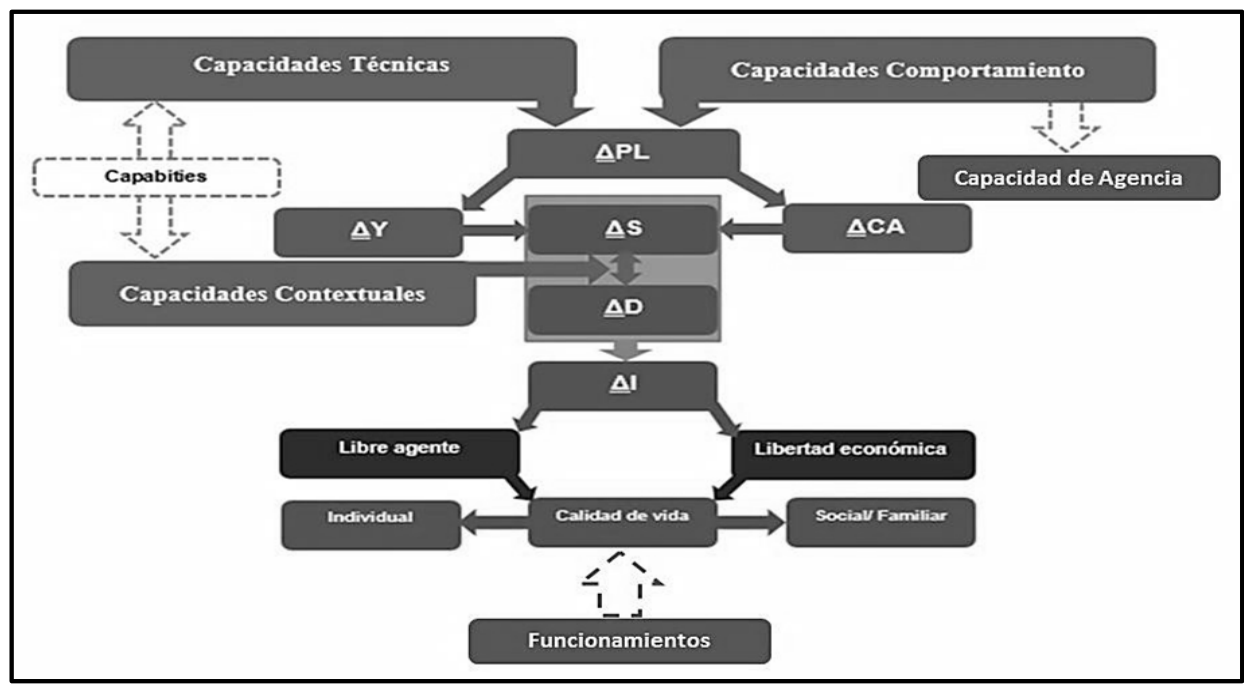

Simbología:

$\triangle P L$ : variación en la productividad del trabajo

$\Delta Y$ : variación en la producción

$\triangle S$ : variación de la oferta

$\triangle C A$ : variación en la curva de aprendizaje

$\triangle D$ : variación en la demanda

$\Delta \mathrm{l}$ : variación del nivel de ingreso

Fuente: Elaboración propia a partir de las bases de datos de GESPLAN-UPM.

En términos generales, el incremento en la producción $\Delta \mathrm{Y}$ y la reducción de costes $\triangle$ CA permitieron a la CMA acrecentar su capacidad para trazar mayor volumen de producción en el mercado. En otras palabras, pudieron incrementar su oferta $(\Delta S)$. 
Todavía quedaba un asunto por resolver: la demanda $(\Delta \mathrm{D})$. El proceso de vertebración entre la $\Delta \mathrm{S}$ y $\Delta \mathrm{D}$ era básico para el logro de los objetivos de la intervención. Para ello, el grupo GESPLAN-UPM, se enfocó en el desarrollo de las capacidades contextuales de las beneficiarias, en procura de aprovechar las condiciones del mercado. En otras palabras, la expansión de las capacidades contextuales permitió la adecuada articulación (Yagüe \& Cazorla, 2013) entre $\Delta \mathrm{S}$ y $\Delta \mathrm{D}$ (ver Figura 2 ).

La articulación entre $\Delta \mathrm{S}$ y $\Delta \mathrm{D}$ redundó en un mayor volumen de ventas (transacciones), y por consiguiente, mayores beneficios para las integrantes de la CMA, incrementando la disponibilidad de ingresos (Sastre-Merino, 2014), así como su capacidad de agente (Nussbaum \& Sen, 1996). Como consecuencia del incremento en el volumen de transacciones, se incrementaron las libertades económicas de las beneficiarias, mejorando no solo sus condiciones de vida y las de sus familiares (ver Figura 2).

\section{CONCLUSIONES}

En el pensamiento de Amartya Sen las libertades constituyen tanto el fin como el medio que impulsa a los pueblos a recorrer los senderos del desarrollo. En términos generales, Sen introduce esta noción como método alternativo para la valoración del desarrollo, centrándose en el proceso de expansión de capacidades (capabilities) para llevar a cabo el tipo de vida que las personas valoran (functioning).

Su enfoque de Development as Freedom es una potente herramienta teórica que permitió la evaluación del Proyecto mediante el uso de los conceptos derivativos de capabilities, Functioning y libre agencia.

Se ha mostrado cómo el proyecto ha contribuido a la expansión de las libertades de las beneficiarias. Estas libertades se han acrecentado básicamente por tres razones: la potenciación de capabilities como resultado del incremento de las capacidades técnicas (fundamentalmente), contextuales y de comportamiento; al acrecentamiento en la dimensión contextual de los functioning, expresado a través de la mejora en la alimentación y educación de los dependientes de las beneficiarias; y por último, en el aumento de la capacidad de agencia en las dimensiones técnicas, contextuales y de comportamiento, reflejado a través del incremento en el liderazgo, la asunción de funciones administrativas y contables, pero sobre todo, el incremento en los niveles de ingreso que posibilitó un mayor número de transacciones.

El proyecto impulsó la generación de capacidades a través de procesos de tecnificación, potenciando la productividad laboral y contribuyendo así, a la hechura de un producto de mayor calidad, y consecuentemente, de mayor valor agregado. Este proceso generó las condiciones para el aprovechamiento y 
ampliación de las redes de acceso a diferentes mercados, ampliando las opciones de comercialización de las beneficiarias.

Se considera especialmente relevante que el proyecto cohesionara la oferta global de los productos elaborados por la CMA, lo que redunda en un mayor poder de mercado, que unido a la comercialización directa (sin intermediación), devino en un mayor nivel de renta, mejorando la calidad de vida de las beneficiarias, y en algunos casos las de sus familiares.

Un elemento que tuvo gran repercusión en la expansión de las libertades económicas de las beneficiarias, fueron los acuerdos con otras instituciones, como: DPD, Galerías Î́ndigo, Moda Shopping y Artatlas, que permitieron aprovechar las condiciones contextuales del mercado.

Como respuesta a la intervención, la CMA cambió y transformó su accionar, al pasar de la búsqueda de libertades constitutivas (evangelización, la lucha por los derechos de la mujer y la lucha contra la violencia familiar) a la búsqueda de libertades instrumentales (como el desarrollo personal, grupal, comunal, entre otros). Este cambio de enfoque, fue uno de los mayores impulsores del proceso de expansión de las libertades económicas.

\section{REFERENCIAS BIBLIOGRÁFICAS}

BUTERO, D. (2011). En busca del desarrollo como libertad. Aportes y críticas de Amartya Sen al desarrollo humano. Santander, Colombia: Universidad Insdustrial de Santander.

CAZORLA, A.; DE LOS RIOS, I.; HERNANDEZ, D.; y YAGUE, J. L. (2010). Case Study: Working With People: rural development project with aymaras communities of Peru; Selected Proceedings Congress, Programme, Ref-348. Ageng, International Conference on Agricultural Engineering.

CENSI, A. (actubre de 2011). "Economía, ética y libertad en el enfoque de las capacidades". Revista Laguna, 29: pp. 123-147.

FERNANDEZ, Á.; HUESO, A.; y BONI, A. (11-13 de Julio de 2012). Contribución del enfoque de las capacidades de Sen a los proyectos de Cooperación Internacional. XVI Congreso Internacional de Ingenieria de Proyectos, pp. 1541-1553.

GOUGH, I. (2007). "El enfoque de lascapacidades de M. Nussbaum: un análisis comparado con nuestra teoría de las necesidades humanas". Papeles de Relaciones Ecosociales y Cambio Global(100), pp.176-202.

HORTON, D. (2004). “¿Cómo planificar, implementar y evaluar el desarrollo el desarrollo de capacidades?". Servicio Internacional para la Investigación Agrícola Nacional, I(64): pp. 1-8.

MONTUSCHI, L. (2008). Libertad, el libre albedrío y el enfoque de las capacidades: El Desarrollo Humano como Libertad. (J. M. Streb, Ed.) Universidad del CEMA(372), 131. 
NEGRILLO, X. (2010). Aprendizaje social en los proyectos de desarrollo: El caso de la Coordinadora de Mujeres Aymaras en Juli (Puno, Perú). 14th International Congress on Project engineering, pp. 1683-1699.

NEGRILLO, X.; YAGUE, J. L.; HERNANDEZ, D.; y VILCA, N. (2011). El Aprendizaje Social como modelo de planificación y gestión de proyectos de desarrollo: La Coordinadora de Mujeres Aymaras. XV Congreso Internacional de Ingeniería de Proyectos, pp. 1683-1699.

NIREMBERG, O. B. (2005). Evaluar para la transformación: Innovaciones en la evaluación de programas y proyectos sociales. Buenos Aires, Argentina: Paidos SAICF.

NUSSBAUM, M.; y Sen, A. K. (1996). La calidad de vida (Primera ed.). Ciudad de México, México: Oxford University Press.

PNUD. (2013). Informe de desarrollo humano Perú 2013. Lima: Programa de Naciones Unidas para el Desarro Humano.

ROBEYNS, I. (2005). "The Capability Approach: A Theoretical survey". Journal Of Human Development, 6(1), pp. 93-117.

SASTRE-MERINO, S. (2014). Desarrollo de capacidades para el liderazgo en proyectos de desarrollo rural. Aplicación comunidades aymaras. Madrid: Universidad Politécnica de Madrid.

SASTRE-MERINO, S.; NEGRILLO, S.; y HERNANDEZ, D. (2013). "Sustainability of Rural Development Projects within the Working With People Model: Application to Aymara Women Communities in the Puno Region, Peru". Cuadernos de desarrollo rural, $1 O(70)$, pp. 219-244.

SEN, A. K. (1985). "Well-being, agency and freedom: The dewey lectures 1984". The Journal of Philosophy(82), pp. 169-221.

SEN, A. K. (1986). Los tontos racionales: una crítica a los fundamentos conductistas de la teoría económica (F.C.E. ed.). México: Filosofia y teoría económica.

SEN, A. K. (octubre de 1993). "Markets and freedoms: Achievements and limitations of the market mechanism". Oxford Economic Paper, 45(4), pp. 519-541.

SEN, A. K. (1995). Nuevo examen de la desigualdad. Madrid, España: Alianza Editorial.

SEN, A. K. (1999). Desarrollo y Libertad (Primera Edición ed.). (Alfred A. Knopf Inc., Ed., E. Robasco, \& L. Toharia, Trads.) Barcelona: Planeta, S. A.

TAMAYO, M. (2004). El proceso de la investigación científica (Grupo Noriega Editores ed.). Ciudad de México, México: Limusa, S. A.

URQUIJO, M. (2007). El enfoque de las capacidades de Amartya Sen: alcance y límites. Valencia: Universitat de Valencia.

YAGUE, J. L., y Cazorla, A. (2013). Estudio de caso fortalecimiento de capacidades de las mujeres aymaras en Puno (Perú). Madrid: No publicado. 
